\title{
Applicability of a new specialty pharmacy-reported measure describing completion of therapy for hepatitis $\mathrm{C}$
}

\author{
Clay Bolduc, PharmD; Kenneth McCall III, PharmD, BCGP, FAPhA; Kirsten Stickney, PharmD, CSP; \\ Alycia Gelinas, PharmD, CSP, AAHIVP; and Eileen Levesque, PharmD, CSP
}

\section{What is already known about this subject}

- In 2019, the Utilization Review Accreditation Commission (URAC) required a new reporting measure for specialty pharmacies related to completion of therapy for hepatitis $C$ virus $(\mathrm{HCV})$.

- When selected and taken appropriately, current direct-acting antiviral (DAA) therapies achieve $>90 \%$ sustained virologic response rates.

- Unlike HIV, there is currently no documented standard of adherence to obtain cure rates with DAA regimens.

\section{What this study adds}

- This observational study is one of the first to evaluate a new accrediting body's measure for completion of antiviral therapy for patients with HCV.

- This research addresses an important measurement issue in HCV treatment adherence, and the findings are applicable to quality-of-care measurement and present practical solutions to address the measurement.

\section{ABSTRACT}

BACKGROUND: In 2019, the Utilization Review Accreditation Commission (URAC) required a new reporting measure for specialty pharmacies related to completion of therapy for hepatitis C virus (HCV).

OBJECTIVE: To calculate HCV completion of therapy according to URAC criteria and compare it with a calculation with additional pharmacy proposed adjustments to assess its applicability.

METHODS: This was an observational study of patients who received HCV treatment with a direct-acting antiviral (DAA) from 1 specialty pharmacy. All patients with prescription claims at a pharmacy who had a first fill for a DAA medication between the 2 measurement periods of January 1, 2018-December 31, 2018, and January 1, 2019-December 31, 2019, were included. Additional information was collected via a retrospective chart review and from the pharmacy's electronic medication system. The cumulative gap according to URAC was calculated from claims data by summing the number of days between the last days supply of 1 claim for the prescribed DAA and the subsequent claims. The pharmacy-proposed cumulative gap was calculated using additional information from patient chart notes in order to account for a true start date.

\section{Author affiliations}

Clay Bolduc, PharmD, College of Pharmacy, University of New England, and Apothecary By Design, Portland, ME. Kenneth McCall III, PharmD, BCGP, FAPhA, College of Pharmacy, University of New England, Portland, ME. Kirsten Stickney, PharmD, CSP; Alycia Gelinas, PharmD, CSP, AAHIVP; and Eileen Levesque, PharmD, CSP, Apothecary By Design, Portland, ME.

\section{AUTHOR CORRESPONDENCE:} Kenneth McCall III, 207.221.4155; kmccall@une.edu

J Manag Care Spec Pharm 2021;27(2):263-67

Copyright $($ 2021, Academy of Managed Care Pharmacy. All rights reserved.

RESULTS: A total of 1,485 patients were identified as having a first fill of a DAA between the 2 measurement periods. The HCV completion of therapy measure calculated per the URAC definition was $83.4 \%$ in 2018 and $86.5 \%$ in 2019 . The only variable significantly associated with a >15-day gap according to the URAC definition was if the first DAA order was delivered to the prescriber's office instead of the patient's home for 2018 ( $\chi 2$ $[1, N=573]=16.8, P<0.001)$ and 2019 ( $\chi 2[1$, $\mathrm{N}=836]=12.6, P<0.001)$. Using the pharmacyproposed adjustment, the modified HCV completion rates for 2018 and 2019 were $88.9 \%$ and $89.9 \%$, respectively. 
CONCLUSIONS: The accrediting body's definition of completion of therapy may report a falsely high rate of gaps in HCV therapy due to not accounting for the actual DAA start date. This information may prove beneficial for the accrediting body, as it reviews its initial definition of the HCV completion of therapy measure.

Hepatitis C virus (HCV) is one of the 5 main types of hepatitis and has an estimated worldwide prevalence of about 170 million people., ${ }^{1,2} \mathrm{HCV}$ is a blood-borne virus existing in 7 distinct genotypes with various subtypes having geographically different prevalence rates. ${ }^{1,3}$ Once the virus is inside the human body it invades the hepatocytes where it can carry out replication. ${ }^{1,2}$ Acute infection routinely presents asymptomatically with a possibility of host clearance. The remaining $50 \%-80 \%$ of patients who do not spontaneously clear the virus will progress to a chronic infection that, when left untreated, will eventually lead to cirrhosis and an increased risk of hepatocellular carcinoma, liver failure, and liver transplant.,

The goal of treatment for $\mathrm{HCV}$ is viral cure, which is measured via a sustained viral response (SVR) at 12 weeks posttreatment completion. ${ }^{4}$ Treatment for HCV has evolved since the early 1990s with interferon therapy, the addition of ribavirin in 1998 to improve SVR, to the first directacting antivirals (DAA) boceprevir and telaprevir in 2011. ${ }^{1}$ Treatment courses of bocepravir and telaprevir were indicated for $\mathrm{HCV}$ in combination with rivavirin and interferon and remained poorly tolerated. In 2013, sofosbuvir was approved for use in certain genotypes in combination with ribavirin without the need for interferon. In the last several years, additional combination products have been approved by the U.S. Food and Drug Administration that have not only expanded the interferon-free treatment options but also are pangenotypic.

The curative treatment goal differs from other viral infections such as hepatitis B or HIV that have a goal of viral suppression but not complete elimination. ${ }^{1}$ It has been documented for viral suppression of HIV, that patients with high levels of adherence (defined as taking their antiretroviral therapy $>95 \%$ of the time) obtain undetectable viral loads a greater proportion of the time. ${ }^{5,6}$ When selected and taken appropriately, current DAA therapies achieve $>90 \%$ SVR12 rates. ${ }^{3}$ Unlike HIV, there is currently no documented standard of adherence to obtain cure rates with the DAA regimens. Adherence rates in clinical trials have ranged from $84 \%-98 \%$, while real-world reported adherence rates have ranged from $50 \%-100 \% .^{7-10}$
To maintain accreditation, specialty pharmacies are required to report on certain measures. New to these measures for 2019 was the measurement of completion of therapy for HCV when treated with DAA therapy. The measurement, used by the Utilization Review Accreditation Commission (URAC), includes all patients in the denominator who were aged 18 years and older with a first fill for a DAA during the specified measurement period. ${ }^{11,12}$ Per the accrediting body's definition, there are 2 cases in which patients are excluded from the numerator but remain included in the denominator. The first case is patients who develop a $>15$-day cumulative gap between their first and last fills of the DAA. ${ }^{11}$ The second case is patients who do not meet the accrediting body's definition of minimum duration of therapy. ${ }^{1}$

The URAC definition of minimum duration of therapy is the minimum length of therapy approved for a specific DAA to treat HCV regardless of the patient's clinical picture. Notably, this definition does not account for patients with $\mathrm{HCV}$ infections that may warrant extended treatment due to factors such as presence of cirrhosis, high viral load, and HIV co-infection. . $^{411}$

The objective of this study was to calculate HCV completion of therapy according to new URAC criteria and compare it to a calculation with additional pharmacy proposed adjustments to assess its applicability.

\section{Methods}

An observational, retrospective, cohort study was conducted with patients who received a first fill for a DAA at a specialty pharmacy during the measurement periods of January 1 , 2018-December 31, 2018 and January 1, 2019-December 31, 2019. Patients were identified by a report run by the pharmacy's electronic medication record system that pulled all paid prescription data claims for DAA agents during the measurement periods. Information collected from the pharmacy electronic medication record system report included patient name, DAA agent, quantity filled, refill number, and date of paid claims. Patients whose first DAA agent fill fell outside of the 2 measurement periods were excluded from the study. Additional information was collected via a manual chart review and included age at first fill, gender, location of first delivery, presence of cirrhosis, and $\mathrm{HCV}$ genotype.

The accrediting body's definition of HCV completion of therapy is a calculation with "Patients who received cumulative days supply required to complete the minimum duration of therapy and did not develop a cumulative gap $>15$ days between the first and last fill of the DAA" in the numerator. ${ }^{11}$ The denominator of this calculation is "Patients aged $\geq 18$ years with a first fill for a DAA during the specified 


\begin{tabular}{|c|c|c|}
\hline \multirow{3}{*}{$\begin{array}{l}\text { Average age } \pm \text { SD } \\
\text { Male sex, } n(\%)\end{array}$} & $2018(n=602)$ & $2019(n=883)$ \\
\hline & $51 \pm 15$ & $49 \pm 15$ \\
\hline & $336 \quad(55.8)$ & $525 \quad(59.4)$ \\
\hline \multicolumn{3}{|l|}{ Genotype, n (\%) } \\
\hline 1 & $390 \quad(64.8)$ & $564 \quad(63.9)$ \\
\hline 2 & $89 \quad(14.8)$ & 122 (12.7) \\
\hline 3 & $114 \quad(18.9)$ & $178 \quad(20.2)$ \\
\hline 4 & $7 \quad(1.2)$ & $12 \quad(1.4)$ \\
\hline 5 & $0 \quad(0.0)$ & $\begin{array}{ll}0 & (0.0) \\
\end{array}$ \\
\hline 6 & $2 \quad(0.3)$ & $(0.2)$ \\
\hline Unknown & $2 \quad(0.3)$ & $9 \quad(1.0)$ \\
\hline Cirrhosis, n (\%) & $155 \quad(25.9)$ & $163(18.4)$ \\
\hline
\end{tabular}

was used to compare patients (i.e., genotype, DAA regimen, presence/absence of cirrhosis, and home vs. office delivery) with a cumulative gap to those without using $\alpha=0.05$.

\section{Results}

There were 602 and 883 patients identified for the 2018 and 2019 measurement periods, respectively. A total of 1,485 patients were included in the study population. The average age for the 2 cohorts were 51 (range 21-90) and 49 (range 21-94) years, and both were predominantly male at 336 (56\%) and 564 (59\%), respectively. Genotype 1 was the most common genotype for patients in 2018 (390, 65\%) and 2019 (564, 64\%). Additional patient demographics can be found in Table 1.

Glecaprevir/pibrentasvir (Mavyret) was the DAA prescribed most frequently for 250 (41.5\%) patients in 2018 and 568 (64.3\%) patients in 2019. In 2018, ledipasvir/sofosbuvir (Harvoni) was the second most commonly prescribed DAA $(180,29.9 \%)$ followed by sofosbuvir/velpatasvir (Epclusa), which was prescribed for 155 patients (25.7\%). Whereas in 2019, sofosbuvir/velpatasvir was the second most commonly prescribed DAA (211, 23.8\%) followed by ledipasvir/ sofosbuvir, which was prescribed for 92 patients (10.4\%).

The HCV completion of therapy rate according to the accrediting body's definition in 2018 was 502 of 602 patients (83.4\%). Of the 100 patients excluded from the numerator in 2018 per the URAC definition, 47 patients were transferred to another pharmacy provider; 19 patients were unable to be serviced (i.e., lost to follow-up, never began treatment, or unable to finish treatment); and 34 patients had a cumulative gap $>15$ days. In 2019, the calculated completion rate according to the accrediting body's definition was 764 of 883 patients (86.5\%). Of the 119 patients excluded from the numerator in 2019 per the URAC definition, 40 (34\%) patients were transferred to another pharmacy provider; 46 (39\%) patients were unable to be serviced; and 33 (28\%) patients had a cumulative gap $>15$ days.

Of the 602 patients in 2018, the first DAA prescription order was delivered to the patient's home, delivered to the prescriber's office, or picked up from the pharmacy for 303 (50.3\%), 270 (44.9\%), and 29 (4.8\%) patients, respectively. However, among the 34 patients who had a cumulative gap $>15$ days according to URAC in 2018, the first DAA prescription order was delivered to the prescriber's office $(27,79.4 \%)$ more often than delivered to the patient's home $(6,17.6 \%)$. Of the 883 patients in 2019, the first DAA prescription order was delivered to the patient's home, delivered to the prescriber's office, or picked up from the pharmacy for 475 (53.8\%), 361 (40.9\%), and 47 (5.3\%) patients, respectively. However, among the 33 patients who had a cumulative gap 


\section{TABLE 22018 and 2019 Sample Characteristics with Chi-Square Analysis to Examine Differences in the Development of a Cumulative Gap}

\begin{tabular}{|c|c|c|c|c|}
\hline Characteristics & $\begin{array}{c}\text { >15-Day } \\
\text { Cumulative } \\
\text { Gap }\end{array}$ & $\begin{array}{c}\leq 15-\text { Day } \\
\text { Cumulative } \\
\text { Gap }\end{array}$ & $\chi^{2}(\mathrm{df})$ & P Value \\
\hline \multicolumn{5}{|l|}{ Cirrhotic (2018) } \\
\hline Yes & 6 & 149 & \multirow{2}{*}{$1.25(1)$} & \multirow{2}{*}{0.26} \\
\hline No & 28 & 418 & & \\
\hline \multicolumn{5}{|l|}{ Genotype (2018) } \\
\hline 1 & 20 & 370 & \multirow{3}{*}{$1.05 \quad(2)$} & \multirow{3}{*}{0.59} \\
\hline 2 & 7 & 82 & & \\
\hline 3 & 7 & 107 & & \\
\hline \multicolumn{5}{|l|}{ DAA (2018) } \\
\hline glecaprevir/pibrentasvir & 13 & 237 & \multirow{4}{*}{$2.59 \quad(3)$} & \multirow{4}{*}{0.46} \\
\hline sofosbuvir/velpatasvir & 6 & 149 & & \\
\hline ledipasvir/sofosbuvir & 14 & 166 & & \\
\hline voxilaprevir/velpatasvir/sofosbuvir & 1 & 13 & & \\
\hline \multicolumn{5}{|l|}{ Cirrhotic (2019) } \\
\hline Yes & 8 & 155 & \multirow{2}{*}{$0.76 \quad(1)$} & \multirow{2}{*}{0.38} \\
\hline No & 25 & 695 & & \\
\hline \multicolumn{5}{|l|}{ Genotype (2019) } \\
\hline 1 & 19 & 545 & \multirow{4}{*}{$2.96 \quad(3)$} & \multirow{4}{*}{0.40} \\
\hline 2 & 7 & 115 & & \\
\hline 3 & 6 & 172 & & \\
\hline Unknown & 1 & 8 & & \\
\hline \multicolumn{5}{|l|}{ DAA (2019) } \\
\hline glecaprevir/pibrentasvir & 23 & 545 & \multirow{3}{*}{$2.08 \quad(2)$} & \multirow{3}{*}{0.35} \\
\hline sofosbuvir/velpatasvir & 9 & 202 & & \\
\hline ledipasvir/sofosbuvir & 1 & 91 & & \\
\hline
\end{tabular}

$\mathrm{x} 2=$ chi square; $D A A=$ direct-acting antiviral; $d f=$ degrees of freedom.

$>15$ days according to URAC in 2019, the first DAA prescription order was delivered to the prescriber's office (23, $69.7 \%)$ more often than delivered to the patient's home $(8,24.2 \%)$.

Among the patients who developed a cumulative gap of $>15$ days according to URAC (34 in 2018 and 33 in 2019), the only statistically significant factor associated with a gap was if the first prescription order was delivered to the prescriber's office instead of the patient's home for 2018
$\left(X^{2}[1, N=573]=16.84, P<0.0001\right)$ and $2019\left(X^{2}[1, \mathrm{~N}=836]=12.6, \quad \mathrm{P}<0.001\right)$. There were no statistically significant differences in either measurement year for the development of a cumulative gap for the other characteristics listed in Table 2. When the true start date for the new DAA regimen was identified by chart review, only 1 of the 34 patients in 2018 and only 3 of the 33 patients in 2019 were confirmed to actually have a gap in therapy of $>15$ days. Thus, 33 additional patients in 2018 and 30 additional patients in 2019 completed therapy without a gap. Adding these patients back into the numerator in a pharmacy proposed adjustment resulted in a modified HCV completion rate of 535 of 602 (88.9\%) patients (compared with the $83.4 \%$ reported) in 2018 and 794 of 883 (89.9\%) patients (compared with the 86.5\% reported) in 2019.

\section{Discussion}

The accrediting body's current definition of HCV completion of therapy does not accurately reflect the actual rates of completion of therapy taking place at this specialty pharmacy. Many specialty pharmacies deliver the first fill of a new therapy via mail to either the prescriber's office or the patient's home. As a result, the date in which a paid prescription claim is generated is not actually when the medication is physically obtained by the patient. This means the accrediting body's completion of therapy measure (which begins on the claim date) identified 63 patients as having gaps in therapy that did not truly experience this therapy interruption.

The only statistically significant finding between the cohorts of patients with and without a cumulative gap $>15$ days according to the URAC definition was the location of the first shipment of medication. Some medical offices require either the first or all shipments be sent to their office for their staff to perform additional medication counseling. The documented DAA start date may differ from the paid claim date when the medication was delivered to the prescriber's office rather than directly to the patient. As a result, there is the potential for the patient's medication to be housed at the office for an extended period of time before the patient is able to be scheduled to come into the office and pick up the medication. This can 
result in the patient being identified as having a gap in therapy per the accrediting body's definition before they have even started therapy. This issue is related to pharmacy claims processing and may vary depending on the pharmacy's clinic coordination strategies.

Additionally, per the accrediting body's definition of minimum duration of therapy, all patients who do not complete all required fills from the same pharmacy are viewed as not completing therapy. In 2018, there were 47 patients who had their prescriptions either transferred as mandated by their insurance plans or received medication from either the drug manufacturer or their prescribers' offices. Similarly, in 2019, there were 40 patients who had their prescriptions transferred due to insurance requirements. The accrediting body's definition identified 81 patients in 2018 and 77 patients in 2019 as being unable to complete their $\mathrm{HCV}$ regimens appropriately due to these factors, when in reality, many of them were likely to have finished their therapy in its entirety. The definition of minimum duration of therapy may need further clarification from URAC as to ensure a uniform interpretation of the criteria.

The pharmacy-proposed definition aims to correct what we consider to be inappropriately calculated gaps by using notes entered into the pharmacy's electronic record system. It would also be reasonable to either eliminate transferred patients from the rate altogether or to include them in the numerator as having completed treatment with another pharmacy.

\section{LIMITATIONS}

This study has several limitations, and results should be interpreted with caution. First, the accrediting body's definition and the pharmacy's proposed definition makes the assumption that if the patients have the medication on hand, they are taking it as prescribed. Second, although the cohort is nearly 1,500 patients, it still only represents 1 specialty pharmacy. Also, the findings were not multivariable adjusted for differences in characteristics between patients. Finally, this was an observational study that cannot control for all of the other unmeasurable factors in patients treated with DAA therapy for $\mathrm{HCV}$.

\section{Conclusions}

The accrediting body's definition of $\mathrm{HCV}$ completion of therapy is a more conservative percentage than what is calculated by the pharmacy's proposed rate. In addition, the accrediting body may be inappropriately eliminating patients from the numerator based on their exclusion criteria, especially patients who had a delay in treatment initiation following the first fill. Further studies at additional specialty pharmacies are needed to confirm results.

\section{DISCLOSURES}

No outside funding supported this study. Levesque reports participation in AbbVie's speaker's bureau with regard to its immunology portfolio. The other authors have no possible financial or personal relationships with commercial entities to disclose that may have a direct or indirect interest in the matter of this study.

\section{REFERENCES}

1. Wang LS, D'Souza LS, Jacobson IM. Hepatitis C-a clinical review. J Med Virol. 2016;88(11):1844-55.

2. Li HC, Lo SY. Hepatitis C virus: virology, diagnosis and treatment. World J Hepatol. 2015;7(10):1377-89.

3. Spengler U. Direct antiviral agents (DAAs)-a new age in the treatment of hepatitis $\mathrm{C}$ virus infection. Pharmacol Ther. 2018;183:118-26.
4. AASLD-IDSA. Recommendations for testing, managing, and treating hepatitis C. Accessed January 6, 2021. http://www. hcvguidelines.org

5. Zaepfel M, Cristofaro L, Trawinski A, et al. Evaluation of a hepatitis C patient management program at a university specialty pharmacy. Ann Pharmacother. 2017;51(4):307-14.

6. Butt AA, Yan P, Shaikh OS, Chung RT, Sherman KE. Treatment adherence and virological response rates in hepatitis $\mathrm{C}$ virus infected persons treated with sofosbuvir-based regimens: results from ERCHIVES. Liver Int. 2016;36(9):1275-83.

7. Mannheimer S, Friedland G, Matts J, et al. The consistency of adherence to antiretroviral therapy predicts biologic outcomes for human immunodeficiency virus-infected persons in clinical trials. Clin Infect Dis. 2002;34(8):1115-21.

8. Low-Beer S, Yip B, O'Shaughnessy MV, et al. Adherence to triple therapy and viral load response. J Acquir Immune Defic Syndr. 2000;23(4):360-61.

9. Grebely J, Mauss J, Brown A, et al. Efficacy and safety of ledipasvir/sofosbuvir with and without ribavirin in patients with chronic HCV genotype 1 infection receiving opioid substitution therapy: analysis of the phase 3 ION trials. Clin Infect Dis. 2016;63(11):1405-11.

10. Younossi ZM, Stepanova M, Henry L, et al. Adherence to treatment of chronic hepatitis C: from interferon containing regimens to interferon and ribavirin free regimens. Medicine (Baltimore). 2016;95(28):e4151.

11. URAC. 2019 URAC specialty pharmacy performance measurement: aggregate summary performance report. December 2019. Accessed January 15, 2021. https:// mk0uracwebqjdtrmplxc.kinstacdn. com/wp-content/uploads/2020/12/ URAC-Specialty-Pharmacy-AggregateSummary-Report-December-2019-1.pdf

12. Pharmacy Quality Alliance. Treatment of chronic hepatitis C - completion of therapy measure (specialty). EQuIPP. Accessed January 15, 2021. https://www. pharmacyquality.com/wp-content/ uploads/2019/01/HepConepagerFINAL.pdf 